Association for Information Systems

AIS Electronic Library (AISeL)

Wirtschaftsinformatik Proceedings 2005

Wirtschaftsinformatik

February 2005

\title{
Erfahrungswissen sichern und aufbereiten - Zur effizienten Gestaltung von Wissensmanagementprozessen bei der BMW AG im Projekt "Werksaufbau Leipzig"
}

Bärbel Fürstenau

Technische Universität Dresden

Johanna Langfermann

Technische Universität Dresden

Fritz Klauser

Universität Leipzig

Volker Born

Universität Leipzig

Follow this and additional works at: http://aisel.aisnet.org/wi2005

\section{Recommended Citation}

Fürstenau, Bärbel; Langfermann, Johanna; Klauser, Fritz; and Born, Volker, "Erfahrungswissen sichern und aufbereiten - Zur effizienten Gestaltung von Wissensmanagementprozessen bei der BMW AG im Projekt "Werksaufbau Leipzig"'" (2005).

Wirtschaftsinformatik Proceedings 2005. 53.

http://aisel.aisnet.org/wi2005/53

This material is brought to you by the Wirtschaftsinformatik at AIS Electronic Library (AISeL). It has been accepted for inclusion in Wirtschaftsinformatik Proceedings 2005 by an authorized administrator of AIS Electronic Library (AISeL). For more information, please contact elibrary@aisnet.org. 
In: Ferstl, Otto K, u.a. (Hg) 2005. Wirtschaftsinformatik 2005: eEconomy, eGovernment, eSociety; 7. Internationale Tagung Wirtschaftsinformatik 2005. Heidelberg: Physica-Verlag

ISBN: 3-7908-1574-8

(C) Physica-Verlag Heidelberg 2005 


\title{
Erfahrungswissen sichern und aufbereiten - Zur effizienten Gestaltung von Wissensmanagementprozessen bei der BMW AG im Projekt „Werksaufbau Leipzig“
}

\author{
Bärbel Fürstenau, Johanna Langfermann \\ Technische Universität Dresden
}

Fritz Klauser, Volker Born

Universität Leipzig

\begin{abstract}
Zusammenfassung: Während des Aufbaus eines neuen Automobilwerks sammeln die Mitarbeiter und Führungskräfte umfangreiches Erfahrungswissen, das genutzt werden kann, um die Unternehmensprozesse optimal zu gestalten und bei künftigen Projekten Fehler zu vermeiden. Bisher wird die systematische Sicherung und Aufbereitung von Erfahrungswissen allerdings vielfach vernachlässigt. Dies $u$. a. deshalb, weil in Theorie und Praxis nur wenige Ansätze existieren, um Projekterfahrungen zu sichern und für künftige Verwendungen zielgerichtet zur Verfügung zu stellen. Dieses Defizit greift der vorliegende Beitrag am Beispiel der inhaltlichen und strukturellen Gestaltung von Lessons Learned auf. Dabei geht es vor allem darum, die bei der BMW AG im Projekt „, Werksaufbau Leipzig “ entstehenden Lessons Learned für künftige Verwender aufzubereiten und, über bestehende technische Ansätze zur Erschließung von Erfahrungswissen hinausgehend, nachfragerorientiert zu gestalten. Lerneffekte sollen erzielt und organisationale Veränderungsprozesse gefördert werden.
\end{abstract}

Schlüsselworte: Wissensmanagement, Projekt, Automobilindustrie, Erfahrungswissen, Lessons Learned, Dokumentation, Prozessunterstützung

\section{Problemstellung}

Der Umgang mit der Ressource Wissen ist eine zentrale Herausforderung für die Automobilindustrie, wie das Fraunhofer Institut für Arbeitswirtschaft und Organisation in einer neueren Studie feststellte $\left[\mathrm{Bu}^{+} 03\right]$. Die Gestaltung von Wissensmanagementprozessen ist dementsprechend eine strategische Aufgabe in Automobilwerken. 
Im Projekt „Aufbau des BMW Werks Leipzig“ entstehen umfangreiche Erfahrungsbestände zu Verfahren der Problembearbeitung, zu optimierten Prozesslösungen und zu strukturellen Funktionen des Werks. Um dieses beim Werksaufbau entstandene Wissen und Können wiederzuverwenden und um künftige Prozesse zu optimieren, werden die Erfahrungsbestände der Mitarbeiter und Führungskräfte des Aufbauteams in Erfahrungsberichten, sog. Lessons Learned ${ }^{1}$, festgehalten. Untersuchungen, die im BMW-Werk Leipzig durchgeführt wurden ${ }^{2}$, zeigen, dass Mitarbeiter und Führungskräfte ihre Projekterfahrungen nur bedingt aussagekräftig dokumentieren. Die erstellten Dokumente entsprechen nicht den Bedürfnissen künftiger Nachfrager bzw. Verwender, Textverständlichkeit und Transparenz sind vielfach defizitär. Ein systematisches Lernen aus den Erfahrungsbeständen wird daher kaum ermöglicht. Es besteht die Gefahr, dass Lessons Learned ihre Funktion zur Prozessunterstützung und Prozessoptimierung sowie zum Lernen aus Erfahrungen nur unzureichend erfüllen.

Der vorliegende Beitrag thematisiert die beschriebene Problematik. Exemplarisch wird dargestellt, welche strukturellen und inhaltlichen Aspekte bei der Dokumentation zu berücksichtigen sind, um eine nachfragerorientierte Verwendung des Wissens und Könnens, der Erfahrungen, zu sichern. Es wird weiterhin aufgezeigt, wie nachfragerorientiert gestaltete Lessons Learned organisationale Veränderungsprozesse unterstützen können.

\section{Wissenssicherung in komplexen Projekten}

\subsection{Bedarf und Praxis}

Der Bau eines Automobilwerks ist „ein einmaliges, zeitlich, sachlich und räumlich begrenztes Vorhaben, das unter Verwendung knapper Ressourcen durchzuführen ist" [Bühn01, S. 631]. Die Sicherung von Erfahrungsbeständen während des Projektes „Werksaufbau“ ist eine komplexe Herausforderung für die Projektleitung sowie für die Mitarbeiter und Führungskräfte des Aufbauteams, und zwar aus folgenden Gründen:

- Organisations- und Prozessstrukturen verändern sich permanent,

Eine Definition des Begriffes Lessons Learned erfolgt unter 3.2.

Der Lehrstuhl für Berufs- und Wirtschaftspädagogik der Universität Leipzig und der Lehrstuhl für Wirtschaftspädagogik der Technischen Universität Dresden führen im Auftrag der BMW AG ein Projekt zum betrieblichen Wissensmanagement im BMW Werk Leipzig durch. Die zitierten Untersuchungen fanden in diesem Rahmen statt. 
- Mitarbeiter kooperieren nicht nur in vertikalen, sondern auch in horizontalen Organisationsstrukturen,

- neue Akteure sind permanent zu integrieren, weil die Belegschaft und die Anzahl der Kooperationspartner (z. B. Zulieferer, Bauunternehmen, Behörden) kontinuierlich bis zum Serienbetrieb des Werks anwachsen,

- die beteiligten Akteure sind im Verlauf der Aufbauarbeit oftmals mit neuartigen Herausforderungen und Problemen konfrontiert,

- Problemstellungen sind zumeist nur interdisziplinär lösbar,

- ein großer Teil der Mitarbeiter arbeitet erstmalig projektorientiert,

- auf erprobte und standardisierte Prozesse kann nicht zurückgegriffen werden und

- bei den Mitarbeitern und Führungskräften existiert kaum ein Bewusstsein für die Gesamtstruktur des Werks.

Der Veränderungs- und Anpassungsdruck an Entwicklungen des Marktes erfordert von Automobilwerken bereits während der Aufbauphase, geplante Strukturen zu überdenken und ggf. zu verändern. Die Erfahrungen, die Mitarbeiter und Führungskräfte während des Werksaufbaus erwerben, haben daher eine besondere Bedeutung für später anlaufende Teilprozesse, den Dauerbetrieb des Werks und künftige vergleichbare Strukturvorhaben (z. B. den Bau weiterer Produktionsstandorte).

In den einzelnen Projekten des Werksaufbaus entsteht umfangreiches Erfahrungswissen

- zur Grundstruktur des Werks (z. B. Struktur der Flächennutzung, Struktur der Kernprozesse),

- zur Planung und Implementation von Verfahrensabläufen,

- zu Anpassungen der Planungsprämissen ${ }^{3}$ und Strukturvorgaben sowie

- zu Planungs- und Umsetzungsentscheidungen und deren Hintergründen.

Nach Freimuth wurde das Potenzial von Projekten für das Wissensmanagement bisher unterschätzt, obwohl sie ,äußerst effektive Formen der organisationalen Wissensproduktion" sind [Frei97, S. 145]. In der Praxis werden Erfahrungen der Planung und Durchführung von Großprojekten vielfach kaum systematisch gesichert und dokumentiert. Schindler [Schi03] konstatiert einen Bedarf zur Entwick-

3 Prämissen sind grundlegende Vorgaben und Richtlinien für betriebliches Handeln. Eine Prämisse des BMW Werks Leipzig ist das tägliche Produktionsziel von 650 Fahrzeugeinheiten. 
lung geeigneter Verfahren und Methoden für die Aufbereitung von Projekterfahrungen durch die Forschung.

\subsection{Sicherung von Erfahrungswissen - Defizite und Folgen}

Die Leitung des BMW Werks Leipzig verfolgt das Ziel, nicht nur zum Abschluss, sondern schon während des Werksaufbaus Erfahrungsbestände zu sichern. In Dokumenten mit der Bezeichnung „Lessons Learned“ werden Erfahrungen aus den einzelnen Prozessen des Aufbauprojektes festgehalten. Lessons Learned werden jeweils zum Abschluss eines Projektmeilensteins (z. B. nach der personellen Besetzung eines Projektteams) erstellt.

Dokumente sind nach Schoop ,jede Art schriftlich fixierter Informationen“ [Scho99]. Sie dienen der Handlungsvor- und -nachbereitung. Durch die Dokumentation werden der innerbetriebliche Informationsfluss formalisiert und relevante Informationen gesichert [KiKu92]. Dokumente unterstützen die Mitarbeiter und Führungskräfte bei der Bearbeitung der anfallenden Aufgaben. Lessons Learned sollen weiterhin künftige Aufbauprozesse unterstützen. Die nutzergerechte Aufbereitung von Lessons Learned ist eine zentrale Voraussetzung für die Wiederverwendbarkeit bereits existierender Erfahrungsbestände und stellt ein Erfolgskriterium für die Generierung neuen Wissens dar. Die effiziente Verwendung der Dokumentation solchen Wissens wird jedoch vielfach dadurch erschwert, dass ${ }^{4}$

- Dokumente der Prozessbeschreibung (planungs-)technisch ausgerichtet sind und die Beschreibung entscheidungsrelevanter Faktoren fehlt,

- die Kontexte von Entscheidungen sowie die Historie von Beschlüssen nicht bzw. nicht fortlaufend dokumentiert werden,

- Strukturen und Prozesse nicht transparent dargestellt werden,

- Dokumente der Prozessbeschreibung autorenbezogen und nicht nutzergerecht erstellt werden,

- relevante Informationen in den Dokumenten nur mit hohem Zeitaufwand für den Leser auffindbar sind und

- den Autoren das Handwerkszeug fehlt, um Prozesse so zu dokumentieren, dass sie zukünftig wiederverwendet werden können.

Abbildung 1 gibt einen Überblick über die zentralen Defizite der Prozessdokumentation.

4 Die im Folgenden aufgeführten Defizite der Dokumentation und ihre Auswirkungen wurden im Rahmen umfangreicher Interviewstudien mit zahlreichen Projektverantwortlichen des Werksaufbaus erhoben. 


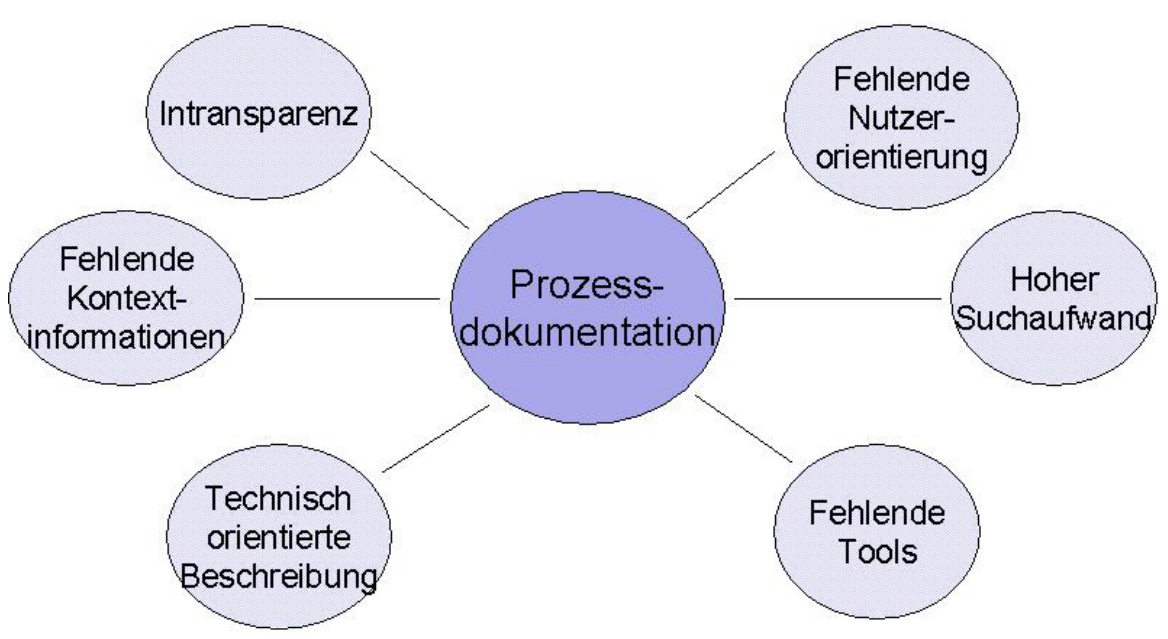

Abbildung 1: Defizite der Prozessdokumentation

Die Folgen dieser Defizite sind, dass

- die Ergebnisse und Erfahrungen aus den Planungs- und Umsetzungsprozessen von (Teil-)Projekten verloren gehen,

- insbesondere die Erfolgsfaktoren für die effiziente Bearbeitung einzelner Prozessphasen nicht identifiziert werden können,

- die Struktur von Prozessabläufen für die Mitarbeiter oftmals intransparent bleibt und

- Teilprojekte nicht oder nur in geringem Maße von Problemlösungen bereits laufender bzw. abgeschlossener Projekte profitieren können.

Zudem können Erfahrungsbestände nicht umfassend für systematisches Lernen genutzt und zur Optimierung der Unternehmensprozesse verwendet werden. In den folgenden Abschnitten wird aufgezeigt, wie diesem Problem durch eine strukturierte Aufbereitung von Erfahrungsbeständen entgegengewirkt werden kann.

\section{Effiziente Sicherung von Erfahrungswissen}

\subsection{Kennzeichen von Erfahrungswissen}

In der Literatur ist nicht einheitlich definiert, was unter Erfahrung bzw. Erfahrungswissen zu verstehen ist. „Oft werden Begriffe wie ,Erfahrungswissen’, ,An- 
wendungswissen', ,Handlungswissen', ,konditionalisiertes Wissen', ,tacit knowledge', ,praktische Intelligenz' oder , praktisches Wissen' in vergleichbarer Weise verwendet" [GrMa96, S. 18]. Dabei steht Erfahrungswissen in engem Zusammenhang mit Expertise. Erfahrung und Expertise haben den gemeinsamen lateinischen Ursprung experior (versuchen, erproben, prüfen) [DaPr98, S. 36]. Experten zeichnen sich durch ein hohes Maß an Erfahrung in einer spezifischen Domäne aus und bringen in dieser dauerhaft herausragende Leistungen [Posn98]. Dabei verfügen sie über eine umfangreiche Wissensbasis, haben überdurchschnittlich großen Erfolg beim Erkennen und Bearbeiten von Problemen, ihre Handlungen sind gekennzeichnet durch hohe Effizienz, Fehlerfreiheit und große Genauigkeit und sie sind besonders flexibel gegenüber neuen Problemstellungen [GrMa96, S. 19].

Erfahrungswissen wird nicht abstrakt, sondern durch Handlungen in konkreten Arbeitssituationen erworben [BüHe03, S. 55]. „Es enthält den für das eigene Handeln bedeutsamen Zusammenhang von Ereignis und Folge in einer Form, wie er für das Handeln unmittelbar nützlich ist" [Fisc96, S. 234]. Erfahrungswissen lässt sich also im Gegensatz zu begrifflich-abstrakt erworbenem Wissen, das oft träge bleibt [Renk96], zur Bewältigung von Situationen anwenden.

\subsection{Lessons Learned: Eine Möglichkeit zur Sicherung und Aufbereitung von Erfahrungswissen}

Die Sicherung und Weitergabe von Erfahrungswissen kann kodifiziert und/oder personifiziert geschehen. Kodifizierung bedeutet, dass die Realität unter Einsatz von Sprache, Logik und Mathematik dokumentiert wird [Boga98, $\mathrm{Ha}^{+} 99$ ]. Im Vordergrund der Personifizierung steht der interpersonelle Wissensaustausch. Damit „Wissen unter den Beteiligten ausgetauscht werden kann“ $\left[\mathrm{Ha}^{+} 99, \mathrm{~S} .87\right]$, ist die Entwicklung von sozialen Netzwerken ${ }^{5}$ in Organisationen unverzichtbar. Im Projekt Werksaufbau der BMW AG ist der Bestand von sozialen Netzen durch den Start des Dauerbetriebs zeitlich begrenzt. Die Projektteams lösen sich in der Regel spätesten zu diesem Zeitpunkt bzw. nach dem Abschluss des jeweiligen Teilprojekts auf, die Mitarbeiter kehren in ihre Fachabteilungen zurück. Die Erfahrungen der Projektmitarbeiter stehen dem Unternehmen danach nur noch mittelbar zur Verfügung. Um die Projekterfahrungen langfristig für das Unternehmen zu sichern, eignen sich Verfahren der Kodifizierung von Wissen, z. B. Lessons Learned.

Lessons Learned haben das Ziel, vergangene Tätigkeiten unter der Perspektive erfolgreicher und weniger erfolgreicher Resultate bzw. Fehler zu dokumentieren und aufzubereiten, um daraus systematisch zu lernen. „Als Ergebnis bündeln Lessons

5 Soziale Netzwerke sind informelle Organisationsstrukturen. Sie geben die tatsächlichen Kommunikations- und Beziehungsstrukturen in Arbeitsabläufen wieder (vgl. Wald 2003). 
Learned in klarer und knapper Form den Kern der kritischen Erfahrungen, die in einem geschäftsrelevanten Feld oder Projekt gemacht wurden $[\ldots]^{“}\left[\operatorname{Re}^{+} 01, \mathrm{~S}\right.$. 115]. Zum einen werden dadurch Projekterfahrungen für die Organisation verfügbar gemacht, zum anderen ergibt sich für das Projektteam während der Laufzeit und zum Projektabschluss ein Lerneffekt, der für die Arbeit in den Fachabteilungen genutzt werden kann. [ $\operatorname{Pr}^{+} 03$, S. 134].

\subsubsection{Analyse von Lessons Learned bei BMW}

Wenn Lessons Learned dazu dienen sollen, Projekterfahrungen zu einem späteren Zeitpunkt nachzuvollziehen, um dadurch bewährte Handlungsweisen übernehmen und Fehler vermeiden zu können, müssen sie im Hinblick auf die potenziellen Bedürfnisse der zukünftigen Nutzer verfasst werden, d. h., Lessons Learned sind didaktisch aufzubereiten.

Ein Problem besteht bei BMW in diesem Zusammenhang darin, dass es keinen definierten Prozess für die Dokumentation von Lessons Learned gibt: Jeder Teilprojektleiter ist für die Sicherung seiner Projekterfahrungen verantwortlich. Wie das geschieht, ist nicht verbindlich geregelt. Handlungsleitend ist lediglich die Fragestellung: „Wie wäre ich effizienter zum Ziel gekommen?“ Dabei werden die Mitarbeiter durch einen Katalog mit Fragen zu Verantwortlichkeiten und zur Akzeptanz der Aufgaben im Projekt, zum Zeitplan sowie zu den eingesetzten Ressourcen unterstützt. ${ }^{6}$ Diese Form der Erfahrungssicherung birgt Schwierigkeiten: Zum einen sind die Mitarbeiter nur unzureichend über den Nutzen dieser Zusatzarbeit informiert, so dass sich ein Akzeptanzproblem ergibt. Zum anderen fehlen vielen Mitarbeitern die Fähigkeiten und Fertigkeiten zu entscheiden, welche Erfahrungen in Zukunft relevant sein könnten und wie detailliert Wissen gesichert werden sollte.

Da gegenwärtig keine Richtlinien für die Gestaltung von Lessons Learned Dokumenten vorliegen, werden die Erfahrungen kaum zielgerichtet und systematisch gesichert. Das Resultat sind Dokumente, die in der Qualität stark variieren. Häufig fehlen Maßnahmen, die zur Zielerreichung eingeleitet wurden, es werden keine Empfehlungen für Folgeprojekte abgeleitet, Erfahrungsträger und Referenzdokumente werden nicht angegeben, Wichtiges wird nicht hervorgehoben (vgl. Abb. 2). 


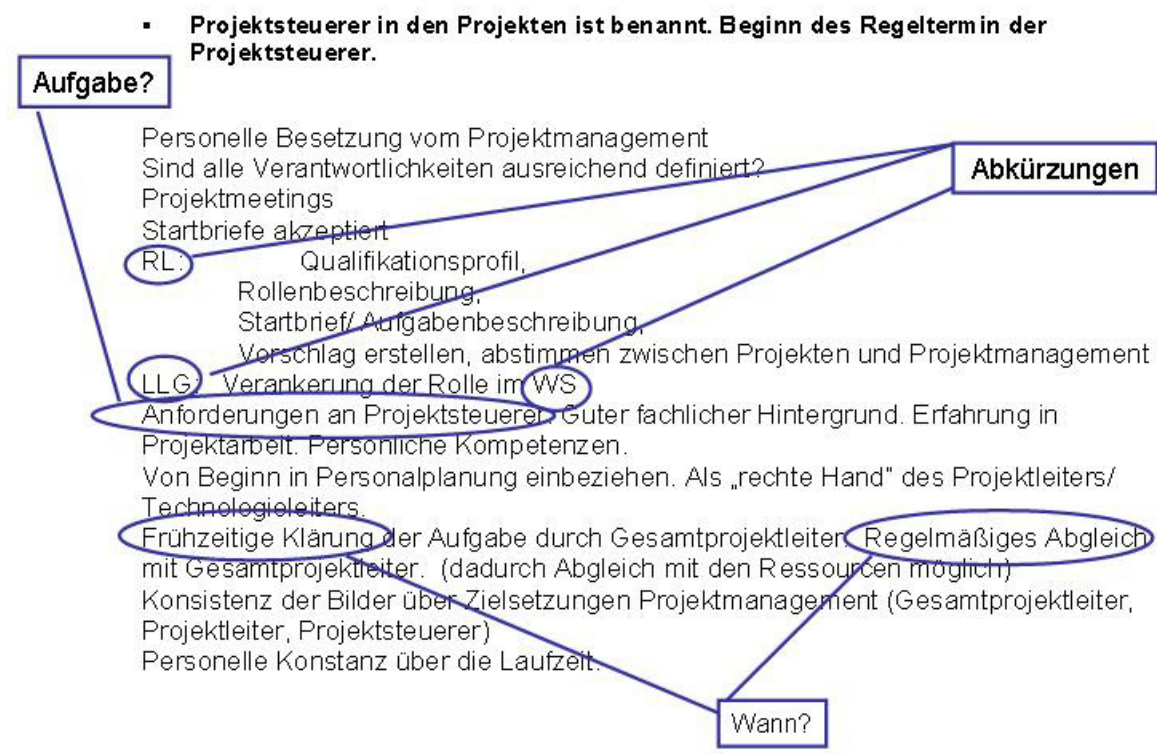

Abbildung 2: Ausschnitt aus einem Lessons Learned Dokument

Durch das uneinheitliche Vorgehen kann sich für künftige Nachfrager das Problem ergeben, dass bedeutsame Informationen nur schwer wiedergefunden und kaum genutzt werden können.

Das Wiederfinden relevanter Erfahrungen wird außerdem dadurch erschwert, dass Mitarbeiter ihre Projekterfahrungen auf Grund fehlender verbindlicher Strukturierungs- und Ausgestaltungsregeln aus einer subjektiven Sichtweise heraus beschreiben oder den Kontext vernachlässigen, in welchem die Erfahrung erworben wurde. In dem abgebildeten Lessons Learned Ausschnitt wird beispielsweise nicht deutlich, worin der Kern der Erfahrung besteht. Es traten offenbar Abstimmungsund Kommunikationsprobleme zwischen den einzelnen Teilprojekten und dem Gesamtprojektmanagement auf. Deshalb wird die Bedeutung des Projektsteuerers als Verbindungsglied zwischen den Schnittstellen betont. Diese Information ist für den Leser jedoch nur nach mehrmaligem Lesen erkennbar.

Verfasser der Lessons Learned verwenden zudem zahlreiche projektinterne Abkürzungen sowie unspezifische und abstrakte Formulierungen, beispielsweise im Hinblick auf Zeitbezüge und Aufgaben (vgl. Abb. 2). Dies hat zur Folge, dass der Nutzer keine konkreten Handlungsanweisungen aus dem Erfahrungsbericht ableiten kann. 
Zusammenfassend ist festzustellen, dass die Mitarbeiter jeweils individuelle Vorgehensweisen für die Sicherung von Projekterfahrungen entwickelt haben. Für eine effiziente und nutzergerechte Dokumentation ist es hingegen notwendig, dass die Dokumente die im folgenden Kapitel genannten Anforderungen erfüllen.

\subsubsection{Anforderungen an die Gestaltung von Lessons Learned}

Ansätze aus der Forschung zum Textverstehen/Textverständnis können einen wichtigen Beitrag für die nachfrageorientierte Strukturierung und Formulierung von Lessons Learned leisten. Langer, Schulz v. Thun und Tausch [Lan 99 ] unterscheiden unter anderem folgende Dimensionen, die Aufschluss über die Formulierung und Strukturierung verständlicher Texte geben:

- Sprachliche Einfachheit: Sprachlich einfache Texte sind gekennzeichnet durch eine einfache Darstellung, kurze und geläufige Sätze und Wörter, erklärte Fremdwörter, konkrete und anschauliche Formulierungen, aktive Verben sowie aktiv-positive Formulierungen.

- Gliederung/Ordnung: Diese Dimension bezieht sich zum einen auf die innere Ordnung und zum anderen auf die äußere Gliederung von Texten. Verständliche Texte sind gegliedert, folgerichtig, übersichtlich, heben wichtige Konzepte hervor, haben einen roten Faden und unterscheiden Wesentliches von Unwesentlichem.

- Kürze/Prägnanz: Mit Hilfe dieses Merkmals lässt sich die Länge des Textes im Verhältnis zum Informationsziel bewerten. Verständliche Texte beschränken sich z. B. auf das Wesentliche und sind auf das Lernziel konzentriert.

Langer et al. [Lan $\left.{ }^{+} 99\right]$ konnten zeigen, dass Texte, die im Hinblick auf diese drei Dimensionen optimiert wurden, besser verstanden und behalten werden.

Neben den o. g. Dimensionen für verständliche Texte, die für die lesergerechte Aufbereitung unterschiedlicher Textsorten herangezogen werden können, müssen Lessons Learned zusätzlich weitere Anforderungen erfüllen, um den spezifischen Bedürfnissen von BMW zu genügen: ${ }^{7}$

- Erfahrungswissen wird in Projekten unter dem Einfluss vieler Faktoren und Rahmenbedingungen generiert und ist nicht losgelöst von diesen zu verstehen. Deshalb ist es wichtig, dass Erfahrungen eingebettet in ihren Kontext dargestellt werden.

- Der Erfahrungsträger sollte über die Projekterfahrungen reflektieren, deren Relevanz für künftige Projekte antizipieren und diese aus einer übergeordneten Perspektive niederschreiben.

7 Die Anforderungen resultieren aus Interviewstudien und Dokumentenanalysen bei der BMW AG. 
- Für den effizienten Zugriff auf relevante Informationen ist entscheidend, dass Erfahrungen zu definierten Themengebieten oder Teilprojekten dokumentiert werden. Zwischen den Themengebieten und Teilprojekten müssen Verknüpfungen bestehen.

- Wichtig ist zudem, dass ähnliche oder vergleichbare Inhalte bezüglich der Formulierung und Darstellung einheitlich behandelt werden, so dass sich Verbindungen zwischen Dokumenten schnell herstellen und Informationen innerhalb eines Dokumentes leicht auffinden lassen.

- Erfahrungen sollen nicht lediglich beschrieben werden, sondern müssen zudem im Hinblick auf ihre Relevanz für Folgeprojekte bewertet werden. Dafür ist es erforderlich, neben den konkreten Erfahrungen Maßnahmen zu dokumentieren, die als Reaktionen eingeleitet wurden.

- Zudem sollte das Ergebnis beschrieben werden, das sich aus den eingeleiteten Maßnahmen ergibt. Die Dokumentation von Referenzdokumenten und Erfahrungsträgern ermöglicht es dem künftigen Nutzer, sich ausführlicher zu den in den Lessons Learned beschriebenen Projekterfahrungen zu informieren.

\subsubsection{Gestaltung von Lessons Learned}

In der Wissensmanagement- und Projektmanagement-Literatur werden verschiedene Methoden zur Sicherung von Projekterfahrungen diskutiert. Viele der beschriebenen Instrumente, wie z. B. der Projektabschlussbericht oder die Projektrevision, fokussieren in erster Linie auf

- die Erfassung standardisierter Kennzahlen,

- die Beschreibung des Projektverlaufs sowie

- die Dokumentation des Projektergebnisses.

Sie vernachlässigen jedoch die Sicherung der Hintergründe von Projekterfahrungen. Die Dokumentation erfolgt hier ausschließlich mit dem Ziel, die Auflösung eines Projektes zu beschreiben [ScEp02, S. 67].

Andere Instrumente stellen die Erhebung von Erfahrungswissen mit Hilfe von Workshops oder Interviews in den Mittelpunkt, geben jedoch meist wenig Hinweise auf die Sicherung und Kommunikation der Erfahrungen im Unternehmen. Von Krogh schlägt beispielsweise die Methode des Projekt-Debriefings vor [vKro98]: Mit Hilfe strukturierter Interviews und Workshops wird das Erfahrungswissen eines Projektteams oder eines einzelnen Mitarbeiters durch einen neutralen, geschulten Dritten, den so genannten Debriefer, erhoben und von ihm dokumentiert. Der Debriefer unterstützt das Projektteam bei der Reflexion über die gewonnenen Erfahrungen und prüft die erhobenen Erfahrungen auf Verständlichkeit. Die Wiedergabe subjektiver, d.h. missverständlicher Sichtweisen, soll damit weitgehend ausgeschlossen werden. 
Auf Grund der engen zeitlichen und personellen Restriktionen in der Endphase des Projektes „Werksaufbau“ ist die Sicherung von Erfahrungen durch aufwändigere Methoden, wie das Projekt-Debriefing, bei der BMW AG derzeit nicht möglich. Es muss daher ein Instrument gefunden werden, das sowohl Hilfestellung bei der inhaltlichen als auch bei der strukturellen Gestaltung von Erfahrungsberichten leistet, so dass der Erfahrungsträger befähigt wird, seine Projekterfahrungen selbständig zu sichern.

Die inhaltliche und strukturelle Gestaltung von Lessons Learned Dokumenten kann über eine Einteilung in jeweils in sich geschlossene Informationseinheiten erreicht werden. Diese sind mit einem Titel zu versehen. Die Titel legen für den Autoren fest, welche Erfahrungen gesichert werden sollen und erleichtern dem Nutzer die Navigation in dem Lessons Learned Dokument.

Mit Hilfe der Information Mapping $®$ Methode [Horn89] können Lessons Learned sowohl inhaltlich als auch strukturell nutzergerecht aufbereitet werden. Information Mapping ${ }^{\circledR}$ basiert auf 30 Jahren wissenschaftlicher Forschung zur Informationsverarbeitung des menschlichen Gehirns und zu Leserreaktionen. Die Methode wurde von dem Kommunikationswissenschaftler und Psychologen Prof. Dr. Robert Horn an der Harvard University, USA, entwickelt, um Studenten ein effektiveres Lernen mit geschriebener Information zu ermöglichen.

Die Methode unterscheidet grundsätzlich zwischen den Informationseinheiten Map und Block. Ein Block ist die kleinste abgeschlossene Informationseinheit und besteht aus einem oder mehreren Sätzen mit einem logisch und sachlich zusammenhängenden Inhalt sowie einem Titel, der den Inhalt des Blocks widerspiegelt. Ein Block enthält einen der sechs für Information Mapping ${ }^{\circledR}$ entwickelten Informationstypen, so dass eine nutzergerechte Aufteilung der Information in kleine Module erreicht wird. Die Informationstypen legen fest, welche Arten von Informationen in einem Block zusammengefasst werden. Mögliche Informationstypen sind Anleitungen, Prinzipien und Fakten. Für jeden Informationstyp werden Empfehlungen über die optimale Darstellungart gegeben. Derart gestaltete Texte sind daher sprachlich einfach gehalten, gegliedert und prägnant. Optisch sind die Blö$c k e$ voneinander durch Linien getrennt. Mehrere Blocks bilden eine Map zu einem abgeschlossenen Thema.

Abbildung 3 zeigt eine mögliche Umsetzung der formulierten Anforderungen für das in Abbildung 2 vorgestellte Lessons Learned Dokument der BMW AG. Die Struktur lehnt sich dabei an die Information Mapping ${ }^{\circledR}$ Methode an. 


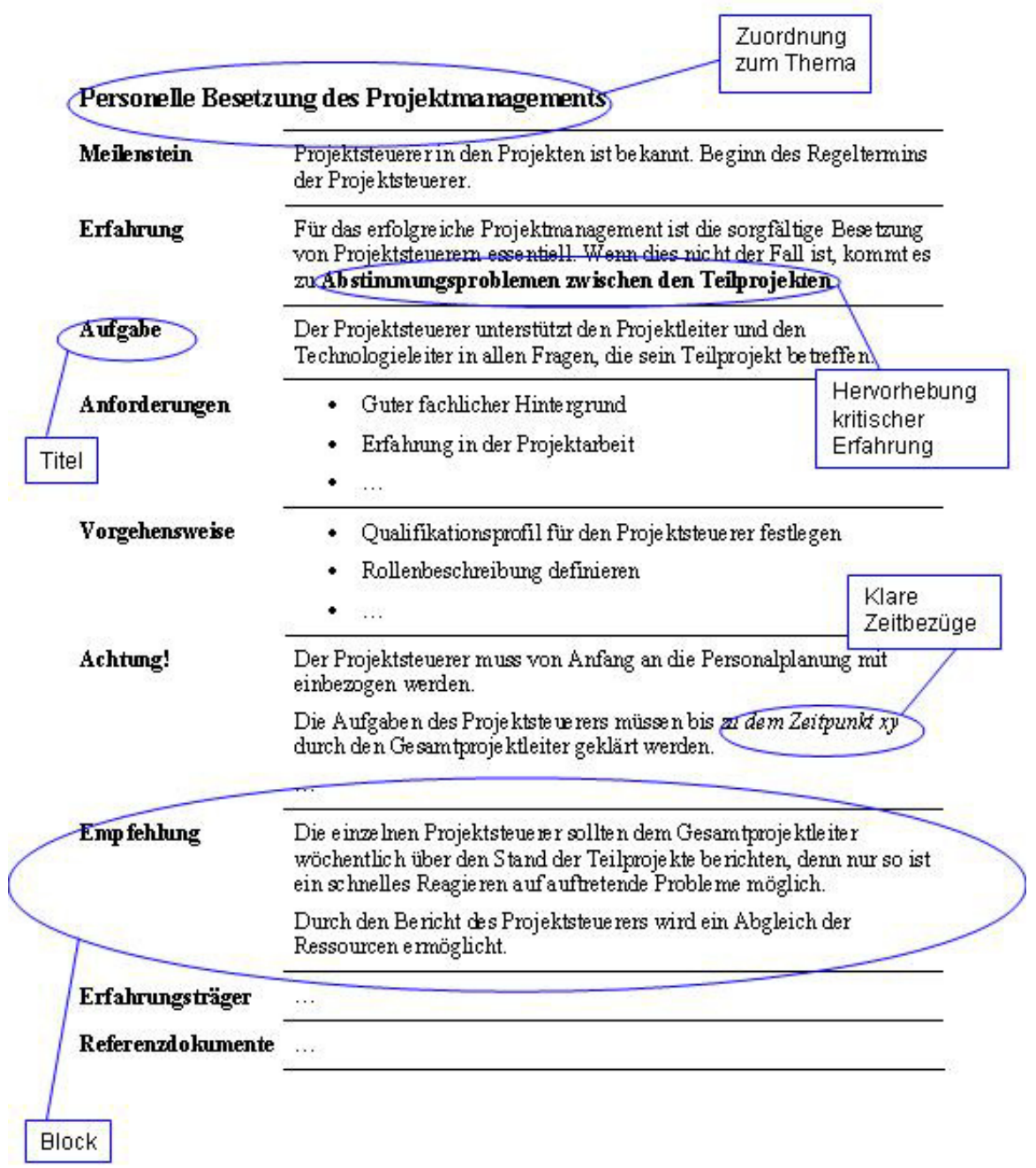

Abbildung 3: Gestaltungsvorschlag für Lessons Learned (Map)

Die vorgestellten Methoden und Verfahren bedürfen einer systematische Fort- und Weiterbildung der Mitarbeiter und Führungskräfte. In einem ersten Schritt erscheinen dafür insbesondere folgende Trainingseinheiten sinnvoll: 
Als Reaktion auf die Probleme, die sich im Hinblick auf die Formulierung von Lessons Learned Dokumenten ergeben, sollte ein Schreibtraining auf der Grundlage von Ergebnissen der Textverstehens-/Textverständnisforschung und der Didaktik entwickelt werden.

Um die Fähigkeiten und Fertigkeiten der Mitarbeiter zur Bewertung der eigenen Projekterfahrungen im Hinblick auf ihre Relevanz für Folgeprojekte zu schulen, ist ein Training zur Förderung metakognitiver Kompetenzen erforderlich.

Zudem sind die Ziele und Vorteile der Sicherung von Projekterfahrungen für die Mitarbeiter transparent zu machen, damit die Akzeptanz zur Dokumentation gefördert wird.

\section{Prozessunterstützung durch Lessons Learned}

Dokumente dienen nicht nur dazu, Wissen und Erfahrungen zu sichern, sondern haben in Unternehmen auch die Aufgabe, betriebliche Prozesse effizient zu gestalten und zu steuern. Sie unterstützen die Mitarbeiter als Funktionsträger im Arbeitsprozess. Insbesondere Lessons Learned Dokumente haben ein hohes Potenzial zur effizienten Prozessunterstützung.

Mitarbeiter werden im Rahmen von Projekten mit Prozessen konfrontiert, die nicht in Handbüchern abgebildet sind. Ziel der Erfahrungssicherung für den Werksaufbau ist es, gerade diese einmaligen Prozesse für die Zukunft zu dokumentieren.

Zudem dienen die in den Lessons Learned dargestellen kritischen Erfahrungen und aufgetretenen Fehler dazu, Prozesse zu optimieren. Vorgehensweisen und Arbeitsabläufe der o. g. Werkserweiterung können ressourcensparender umgesetzt werden. Unternehmensweite Veränderungsprozesse lassen sich effizienter realisieren.

Damit Lessons Learned Funktionen der Prozessunterstützung und -optimierung erfüllen können, werden neben der strukturierten Aufbereitung der Dokumente weitere Anforderungskriterien entwickelt.

Analysiert man die existierende Literatur, die in erster Linie im Bereich der Ingenieurwissenschaft angesiedelt ist, so lassen sich eine Vielzahl unterschiedlicher Einflussfaktoren identifizieren.

Insbesondere der Sicherung von Hintergründen von Entscheidungen und Festlegungen wird eine wichtige Rolle beigemessen [GeWe00, S. 543].

In diesem Zusammenhang wird ein Konzept zur Fortschreibung von Dokumenten von sich verändernden Prozessen entwickelt. Dadurch bleibt ohne aufwändige Su- 
che nachvollziehbar, wie und aus welchen Gründen die Fakten, Festlegungen und Beschlüsse verändert wurden.

Damit „Lessons Learned“ Prozesse beschreiben können, die sich in größeren zeitlichen Abständen wiederholen, erfolgt die Dokumentation so, „dass sich Möglichkeiten ergeben, während praktisch ablaufender Prozesse die tatsächliche Folge von Vorgängen zu dokumentieren." [Pens98, S. 67]. Dies ist wichtig, weil auf diese Weise Abläufe des Werksaufbaus transparent gemacht und die Grundlage für eine Bewertung und Optimierung der Prozesse des Werksaufbaus geschaffen werden können.

Gleichzeitig bietet die Information Mapping® Methode durch die abgeschlossenen Informationsblöcke die Möglichkeit zur arbeitsteiligen Dokumentation. Dies ist bedeutsam, da Prozesse der betrieblichen Leistungserstellung durch arbeitsteilige Vorgehensweisen gekennzeichnet sind [Pens98, S. 71] und nur eine arbeitsteilige Dokumentation gewährleistet, dass der Prozess vollständig abgebildet werden kann.

Zentral für die prozessunterstützende Verwendung der Lessons Learned ist weiterhin, dass die Erfahrungsberichte nicht nur als Ganzes zur Verfügung stehen, sondern dass sie auch in Teilen nutzbar sind. Prozessbeschreibungen sind dazu modular zu gestalten: „Da kein Projektablauf dem anderen gleicht und diese sich vielmehr hinsichtlich Bedeutung, Umfang und Dauer unterscheiden, gewährleistet eine baukastenartige Prozessbeschreibung, dass das Wissen für alle Projekte des Produktionsstandortaufbaus und -anlaufs genutzt werden kann, indem die Module in Abhängigkeit der Planungsaufgabe individuell zusammengefügt werden können." [La+01 S. 366]. Die Abhängigkeiten zwischen den einzelnen Modulen müssen erkennbar bleiben. Auch dies kann durch die beschriebene Methode des Information Mapping erreicht werden.

\section{Fazit und Ausblick}

Zur Zeit wird evaluiert, welche Erfolge durch die genannten Ansätze der strukturellen und inhaltlichen Gestaltung von Lessons Learned erzielt werden können. Erste Rückmeldungen von Projektverantwortlichen verweisen auf eine deutliche Unterstützung der Autoren bei der strukturierten Sicherung und Aufbereitung von Projekterfahrungen durch die vorgestellten Ansätze. Insbesondere das Erfassen wichtiger Kontextinformationen konnte sichergestellt werden. Die Evaluation der Projektergebnisse erfolgt kontinuierlich mit der Implementierung eines Redaktionsprozesses zur Erstellung und Verwendung von Lessons Learned Dokumenten im Rahmen eines umfassenden Feldversuches. Die Evaluationsergebnisse des Feldversuchses werden für weitere Konstruktions- und Implementierungsprozesse nutzbar gemacht. Das betrifft sowohl die Re-Konstruktion projektspezifischer 
Lessons Learned als auch die Entwicklung von Verfahrensregeln zur Durchführung des Redaktionsprozesses. Diese Entwicklungsergebnisse sind in weiterführenden Schulungen zu thematisieren.

Derzeit gibt es vor allem technische Ansätze, Erfahrungen sichtbar und nutzbar zu machen [Stru00; GeWe00]. Die Bedürfnisse der künftigen Nutzer werden darin allerdings vielfach nur in geringem Maße berücksichtigt. Dokumente sind inhaltlich und strukturell so aufzubereiten, dass künftige Nachfrager die dokumentierten Erfahrungen zur Unterstützung von Unternehmensprozessen wieder verwenden können. Hier kann die Wirtschaftspädagogik mit den dargestellen Ansätzen und Methoden einen wichtigen Beitrag leisten und zugleich komplementäre Modelle und Vorgehensweisen zu den technisch orientierten Herangehensweisen entwickeln.

\section{Literatur}

[Be ${ }^{+}$03] Becker, J., Kugeler, M. \& Rosemann, M. (Hrsg.): Prozessmanagement: Ein Leitfaden zur prozessorientierten Organisationsgestaltung. (4.Aufl.). Springer: Berlin; Heidelberg; New York, 2003.

[Boga98] Bogaschewsky, R.: Wissensorientiertes Management. Eine kritische Literaturanalyse. Dresdner Beiträge zur Betriebswirtschaftslehre, Nr. 15/98. Technische Universität Dresden. Fakultät Wirtschaftswissenschaften, 1998.

[Bühn01] Bühner, R. (Hrsg.): Management-Lexikon. Oldenbourg: München, Wien, 2001.

[Bu ${ }^{+}$97] Bullinger, H.-J., Wörner, K. \& Prieto, J.: Wissensmanagement heute. Daten, Fakten, Trends. Stuttgart: Fraunhofer Institut für Arbeitswirtschaft und Organisation, 1997.

[Bu $\left.{ }^{+} 03\right]$ Bullinger, H.-J.; Kiss-Preußinger, E. \& Spath, D.: Automobilentwicklung in Deutschland - wie sicher in die Zukunft? Chancen, Potenziale und Handlungsempfehlungen für 30 Prozent mehr Effizienz. Fraunhofer IRB-Verlag: Stuttgart, 2003.

[BüHe03] Büssing, A.; Herbig, B.: Implizites Wissen und Wissensmanagement - Schwierigkeiten und Chancen im Umgang mit einer wichtigen menschlichen Ressource. Zeitschrift für Personalpsychologie, 2, 2003, S. 51-65.

[DaPr98] Davenport, Th. H. \& Prusak, L.: Das Praxisbuch zum Wissensmanagement. Verlag Moderne Industrie: Landsberg, 1998.

[Fisc96] Fischer, M.: Überlegungen zu einem arbeitspädagogischen und -psychologischen Erfahrungsbegriff. Zeitschrift für Berufs- und Wirtschaftspädagogik, 1996, S. 229-244.

[Frei97] Freimuth, J.: Projektmanagement - Unterschätzte Chance für Personalmanagement und Wissensmanagement. In J. Freimuth, J. Haritz, B.-U. Kiefer (Hrsg.), Auf dem Weg zum Wissensmanagement. Göttingen: Verlag für angewandte Psychologie, 1997, S. $145-155$. 
[GeWe00] Gerken, H. \& Weyrich, M.: Technologien zum Wissensmanagement in der CAD/CAM Prozesskette. Zeitschrift für den wirtschaftlichen Fabrikbetrieb, 95 (11), 2000, S. 543-546.

[Grub99] Gruber, H.: Erfahrung als Grundlage kompetenten Handelns. Huber: Göttingen, 1999.

[GrMa96] Gruber, H. \& Mandl, H.: Erfahrung und Expertise. In H. Gruber \& A. Ziegler (Hrsg.), Expertiseforschung. Theoretische und methodische Grundlagen (S. 18-34). Westdeutscher Verlag: Opladen, 1996.

[Hack99] Hacker, W.: Informationsökonomie aus psychologischer Sicht: Wissensmanagement; Frühes Prototyping bei der Produktentwicklung. Forschungsberichte des Instituts für Allgemeine Psychologie und Methoden der Psychologie der TU Dresden: Band 70. Dresden: TU Dresden, 1999.

[Ha $\left.{ }^{+} 99\right]$ Hansen, M., Nohria, N. \& Tierney, T.: Wie managen Sie das Wissen in Ihrem Unternehmen? Harvard Business Manager. Nr. 5/99, 1999, S. 85-96.

[Horn89] Horn, R.: Mapping Hypertext. Linkage, and Display of Knowledge for the Next Generation of On-Line Text and Graphics. Lexington: Massachusetts, 1998.

[KiKu92] Kieser, A. \& Kubicek, H.: Organisation. de Gryter Verlag: Berlin, 1992.

[Klug99] Kluge, A.: Erfahrungsmanagement in lernenden Organisationen. Verlag für Angewandte Psychologie: Göttingen, 1999.

[vKro98] Krogh, G., von: Care in Knowledge Creation. Califonia Management Review, Volume 40, Number 3, Spring 1998, Haas School of Business, University of Califonia, Berkeley, 1998, pp. 133-153.

[La+ 01] Laik, Th., Hiller, M., Wagenknecht, Ch. \& Warnecke, G.: Erfahrungssicherung bei der Fabrikplanung. Zeitschrift für wirtschaftlichen Fabrikbetrieb, 96 (7-8), 2001, S. 365-368.

[Lan $\left.{ }^{+} 99\right]$ Langer, I., Schulz v. Thun, F. \& Tausch, R.: Sich verständlich ausdrücken. Ernst Reinhardt: München, 1999.

[NoKo98] Nonaka, I. \& Konno, N.: The Concept of „Ba“: Building a Foundation of Knowledge Creation. California Management Review, Volume 40, Number 3. Haas School of Business, University of California, Berkley, 1999, pp. 673-683.

[Pens98] Penschke, St.: Erfahrungswissen in der Produktentwicklung. Erfassung und Aufbereitung prozeßorientierter Informationen in Konstruktionsprojekten. Forschungsbericht VDI Reihe 16, Nr. 98. VDI Verlag: Düsseldorf, 1998.

[Posn98] Posner, M. I.: What is it to be an expert? In M. T. H. Chi, R. Glaser \& M. J. Farr (Eds.), The nature of expertise (pp. 24-36). Erlbaum: Hillsdale, NJ, 1998.

[Pr 03] Probst, G., Raub, St. \& Romhardt, K.: Wissen managen. Wie Unternehmen ihre wertvollste Ressource optimal nutzen. (4. Aufl.). Gabler: Wiesbaden, 2003.

[Re+01] Reinmann-Rothmaier, G., Mandl, H. Erlach, Ch. \& Neubauer, A.: Wissensmanagement lernen. Ein Leitfaden zur Gestaltung von Workshops und zum Selbstlernen. Beltz: Weinheim, 2001. 
[Renk96] Renk1, A.: Träges Wissen: Wenn Erlerntes nicht genutzt wird. Psychologische Rundschau, 47 (2), 1996, S. 78-92.

[Schi03] Schindler, M.: Wissensmanagement in der Projektabwicklung. Eul: Köln, 2003.

[ScEp02] Schindler, M. \& Eppler, M. J.: Vom Debriefing zum kontinuierlichen Erfahrungslernen. Organisationsentwicklung, 1, 2002, S. 58-71.

[Scho99] Schoop, E.: Dokumentenmanagement. In: WISU o. Jg. 10, 2., 1999, S. 1346 1360.

[Stru00] Strunk, H.-M.: Lektion gelernt. Automobilzulieferer nutzt softwareunterstützt FMEA-Wissen für Folgeprojekte. Qualität und Zuverlässigkeit, 45 (5), 2000, S. 574580 .

[Thie01] Thiesse, F.: Prozessorientiertes Wissensmanagement: Konzepte, Methode, Fallbeispiele. St. Gallen: Hochschule für Wirtschafts-, Rechts- und Sozialwissenschaften. Dissertation, 2001.

[Wald03] Wald, A.: Netzwerkstrukturen und -effekte in Organisationen: Eine Netzwerkanalyse in internationalen Unternehmen. Gabler: Wiesbaden, 2003. 
